\title{
Delayed reservation decision in optical burst switching networks with optical buffers
}

\author{
G.M. Li ${ }^{*}$, Victor O.K. $\mathrm{Li}^{+}$ \\ *School of Information Engineering \\ SHANDONG University at WEIHAI, China \\ ${ }^{+}$Department of Electrical and Electronic Engineering \\ The University of Hong Kong, Hong Kong, China
}

\author{
C.Y. Li, P.K.A. Wai \\ Photonics Research Center and Department of Electronic \\ and Information Engineering \\ The Hong Kong Polytechnic University \\ Hong Kong, China
}

\begin{abstract}
The proposed delayed reservation decision scheme offers the same node complexity as that in general OBS networks with optical buffers. Simulations show the proposed scheme has better performance than existing schemes in terms of burst loss probability.
\end{abstract}

\section{Keywords- optical burst switching; Optical Buffer}

\section{INTRODUCTION}

The massive explosion in Internet traffic has driven the development of wavelength division multiplexing (WDM) technology. Typically, the network is circuit-switched, with a lightpath (circuit) set up between the source and destination nodes, and an appropriate wavelength along the route is chosen No optical buffer is needed at intermediate nodes. However, each fiber can only contain a limited number of wavelengths, and if two different lightpaths attempt to share one wavelength in a fiber, one of them must be blocked. Although wavelength converters can be used to decrease the blocking probabilities, the problem of wavelength allocation and routing is NP-hard [1]. Another drawback of wavelength routing is that it is not efficient for bursty data traffic since once a lightpath is set up, the bandwidth resource is dedicated to this lightpath, even if there is no traffic. To improve the bandwidth efficiency, IP over WDM using optical packet switching (OPS) becomes an ideal choice of the next generation networks. Ideally, all functions inside each node of an OPS network are performed in the optical domain [2], i.e., all-optical packet switching. However, optical processing capabilities are very limited and optical random access memory (RAM) is not available at present. In order to accommodate current technology, optical burst switching (OBS) was proposed in the late 1990s [3]. An OBS network consists of edge nodes at the periphery of the network, and core nodes inside the network. Edge nodes aggregate packets from upper layer into optical data bursts (DBs) and keep them in the optical domain. Each optical burst has an associated control packet (CP). CP is sent in a separate control channel and processed electronically $[4,5]$ at each node, in an attempt to schedule its corresponding data burst.

In general OBS networks, nodes processing control packets (CPs) usually follow the first-come-first-served discipline; they can only know about previously scheduled data bursts (DBs), but cannot predict the information of incoming DBs. As a consequence, this leads to inefficient resource utilization [6]. Similarly, although existing OBS networks with optical buffers [7-10] have buffered the CPs with the same delay of the DBs using fiber delay lines (FDLs) in order not to alter the offset between the CPs and the DBs, scheduling the DBs is still based on the arrival sequence of CPs. Thus, resource allocation is inefficient.

In this paper, we propose the delayed reservation decision (DRD) scheme in OBS networks with FDLs to improve the network performance. The remainder of this paper is organized as follows. In the second section, we briefly review optical burst contention resolution using FDLs and then present the proposed scheme. In the third section, we give numerical evaluations and we find that the proposed scheme has better performance than existing schemes. Finally, we conclude.

\section{OBS NETWORKS WITH OPTICAL BUFFERS}

\section{A. General OBS networks with optical buffers}

Although optical buffers are not mandatory in OBS networks, studies show that using FDLs as optical buffers can effectively improve the network performance [7, 10]. In an OBS network with FDLs, when a control packet cannot successfully reserve a wavelength at an output port for its corresponding data burst, it will try to reserve the available FDL with the shortest delay instead. There are two different node structures in OBS networks with FDLs: one is that each link has a dedicated FDL module [9], and we call it FDL share per link; the other is that each node has only one FDL module to be shared by all links [7], and we call it FDL share per node. The difference between FDL share per link and FDL share per node mainly lies in the hardware cost and the efficiency of FDL utilization. FDL share per node needs much less single FDL elements, and much less wavelength converters. But the tradeoff is that it needs more ports in the optical switch. Since wavelength converters are expensive, in terms of minimizing hardware cost, FDL share per node is a better choice since it is less bulky and utilizes FDLs more efficiently than FDL share per link.

To explain the operation of resolving contentions by FDLs, we consider a scenario in which each node has one output port with a single wavelength and a single FDL with fixed delay $T_{d}$, 
as shown in Figure 1. Suppose a new control packet $C P_{x}$ arrives at the current node $i$. $C P_{x}$ contains the information of its offset and its corresponding data burst length information $T_{D B}$. Based on these parameters, the expected data burst arriving time $t_{s t}$ and data burst departing time $t_{e n}\left(t_{e n}=t_{s t}+T_{D B}\right)$ can be obtained. After $C P_{x}$ finishes its processing at time $t_{l}$, it attempts to reserve the wavelength at the output port from $t_{s t}$ to $t_{e n}$. The reservation request for the interval $\left(t_{s t}, t_{e n}\right)$ fails since it is blocked by an already scheduled data burst $D B_{0}$, shown in Figure 1. Then, $C P_{x}$ will check whether contention occurs in the data channel after delay $T_{d}$ and whether the data burst can be accommodated in the FDL with the delay $T_{d}$. If both conditions are satisfied, $C P_{x}$ will reserve the FDL for the interval $\left(t_{s t}, t_{e n}\right)$ at a certain wavelength and reserve the data channel for the interval $\left(t{ }_{s t}, t{ }^{\prime}{ }_{e n}\right)$ simultaneously. When the data burst arrives at node $i$ at time $t_{s t}$, it will go through the FDL. Thus the data burst can be buffered for time $T_{d}$ and the data burst is still kept in the optical domain and transmitted to the downstream nodes.

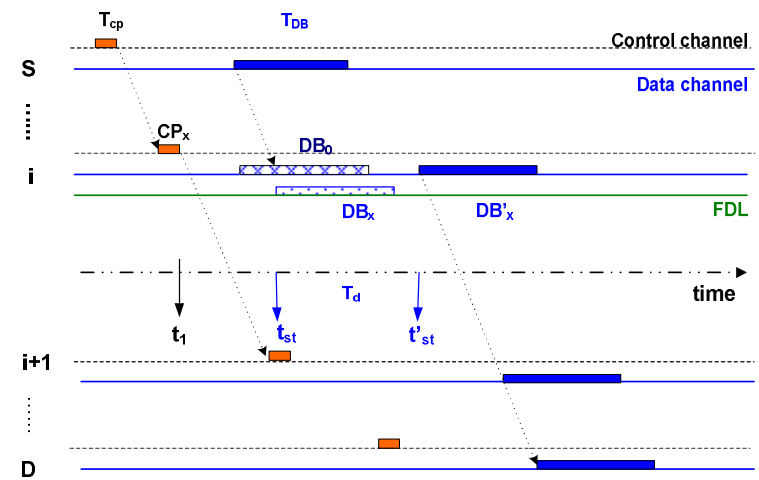

Figure 1. Chronological sequence of a straightforward approach in OBS networks with FDLs; contention occurs at node $i$, the updated control packet is sent out immediately after processing in this scenario.

When FDLs are used as optical buffers to resolve burst contentions in OBS networks, a straightforward option is to send the updated control packet to the downstream nodes immediately after the control packet successfully reserves resources in FDLs and data channel for its corresponding data burst. As shown in Figure 1, after $C P_{x}$ successfully resolves contention using FDL at node $i, C P_{x}$ is updated with the new information of the delayed data burst and sent to the downstream node immediately. The offsets at all downstream nodes will be changed according to the FDL delay at node $i$.

Previous work [4, 7-9] note that a burst with longer offset time has lower dropping probability. FDLs help to improve performance in the extended-offset based quality of service (QoS) provision schemes. Most existing work in OBS networks focus on the general scheme as shown in Figure 2. In order to keep the burst priority unchanged, the corresponding burst offset value must be the same as in the scenario without FDLs. This implies that, after $C P_{x}$ successfully resolves contention using FDL at node $i$, the control packet must be electronically buffered with the same delay as that of the suitable FDL. Under the general reservation scheme in OBS networks with FDLs [7, 8], the decision of the network resource allocation is made immediately after processing the CP. For the example shown in
Figure 2, resource allocation decision is done at $t_{1}$, but $C P_{x}$ has to be electronically buffered with the same delay, $T_{d}$, as the desired FDL; the updated control packet, $C P_{x}$ ', is sent to the down stream node at $t_{l}$. By comparison, the straightforward scheme is more cost-efficient in hardware than the general scheme since it does not need extra electronic buffer for the control packets. Its drawback is that it cannot be implemented to provide quality of service $(\mathrm{QoS})$ in the extended-offset scheme since changing offsets will change the priority of the corresponding data bursts.

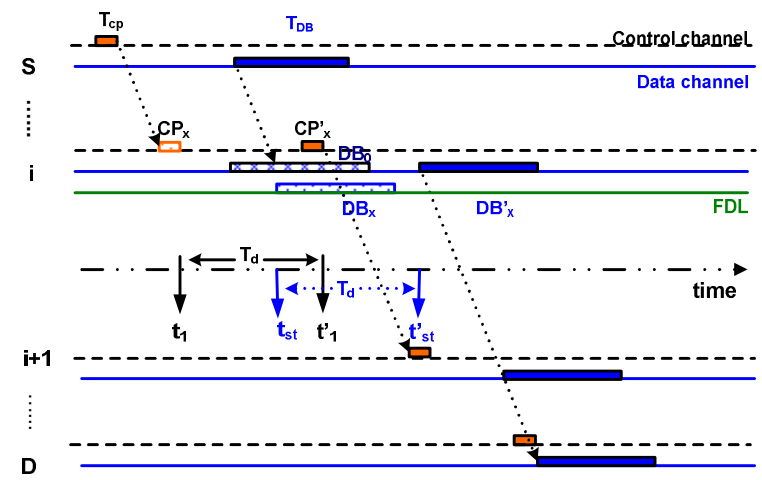

Figure 2. The chronological sequence of burst contention resolution using FDLs in a node with a single wavelength and a single FDL; contention occurs at node $i$, the updated control packet is electronically buffered with $T_{d}$ after processing in this scenario.

\section{B. Delayed reservation scheme}

The first-come-first-served discipline leads to inefficient resource utilization in general OBS networks [6,11]. In most existing work on OBS network with FDLs, control packets are electronically buffered with the same delay as the data bursts using FDLs, and scheduling data bursts in FDLs and data channels is still based on the arrival sequence of control packets. Thus, the resource allocation is still inefficient.

Figure 3 illustrates an example of the inefficient resource allocation in the general OBS networks with FDLs due to the first-come-first-served discipline. We assume each node in a general OBS network has a fiber with a fixed delay $T_{d}$ to resolve burst contentions. We further assume that a node has received three control packets, $c 1, c 2$, and $c 3$, which request the same output port $O_{1}$ with wavelength channels $D 1$ and $D 2$. Their corresponding data bursts are marked $B 1, B 2$, and $B 3$ following their corresponding control packets. $c 1, c 2$, and $c 3$ finish their processing at times $t_{1}, t_{2}$ and $t_{3}$ respectively. We assume that, due to the burst contention at the output port, all three data bursts, $B 1, B 2$, and $B 3$, need to be buffered by the FDL. According to the reservation scheme in general OBS networks with FDLs, control packets $c 1, c 2$ and $c 3$ need to be electronically buffered until $t_{5}, t_{6}$ and $t_{7}$; then their updated corresponding control packets $c 1^{\prime}, c 2^{\prime}$ and $c 3^{\prime}$ are sent to the downstream nodes. The actual time of arrival of $B 1, B 2$, and $B 3$ at the output port are $t_{8}, t_{11}$ and $t_{10}$ respectively. A new incoming control packet, $c 4$, arrives at the node at $t_{4}$ and its data burst $B 4$ arrival time is $t_{9}$ as shown in Figure 3(a).

Follows the common reservation scheme of the general OBS networks with FDLs, the scheduling decisions are made 
as following: $c l$ arrives at the node at $t_{1}$ and its data burst encounters burst contention. $c l$ finds that $B l$ can be scheduled at time $t_{8}$ and there is no burst contention at the desired FDL; thus, after finishing processing $c l$ at $t_{l}$, control packet $c l$ is buffered electronically for an interval $T_{d}=t_{5}-t_{1}$ and the updated control packet $c l^{\prime}$ is sent to the downstream node at $t_{5}$. The delayed data burst is scheduled at $t_{8}$. Control packets $c 2$ and $c 3$ are processed likewise. To minimize the idle periods between data burst transmissions, $B 2$ is scheduled on channel D1 with starting time $t_{11}$ after its control packet finishes processing and makes scheduling decision at $t_{2}$. Consequently, $B 3$ is assigned channel D2 with starting time $t_{10}$ and its scheduling decision is made at $t_{3}$. When $c 4$ arrives at the node, it finds its data burst $B 4$ can not be scheduled on any channel due to the burst contention, as shown in Figure 3(b). Thus, FDLs or other contention resolutions have to be used; otherwise, data burst $B 4$ will be dropped. However, even after delay $T_{d}$, no data channel is available to accommodate the data burst B4, as shown in Figure 3(c). Ultimately, B4 is dropped.
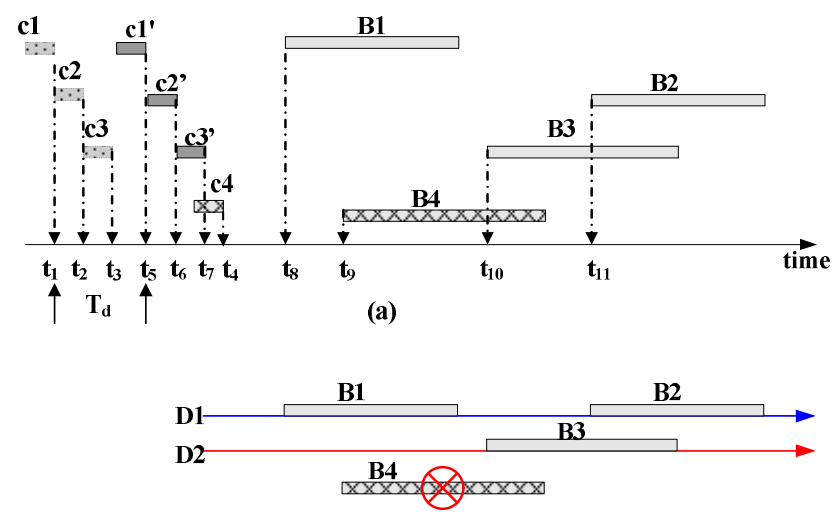

(b)

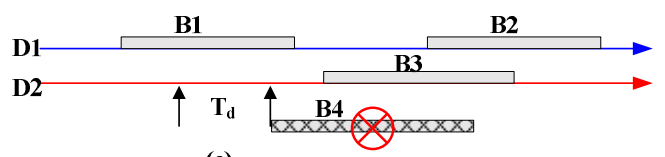

(c)

Figure 3. (a) The arrival and departure sequence of control packets and the corresponding data bursts, (b) channel assignment according to the reservation scheme in general OBS with FDLs; the new incoming data burst cannot be scheduled directly, (c) the new incoming data burst cannot be scheduled after delay $T_{d}$

Under the general reservation scheme in OBS networks with FDLs, we notice that, after control packets successfully resolve contention using FDLs, they have to be electronically buffered with the same delay as the selected FDLs. In fact, the decision of the network resource allocation is made immediately after processing the control packet. The process of electronic buffering does not contribute to the network performance. Hereby, we propose the delayed reservation decision (DRD) scheme.

The proposed scheme takes advantage of the process of electronic buffering of control packets with the aim to improve the resource utilization efficiency. As shown in Figure 2, under the proposed scheme, rather than making resource allocation decision after processing $C P_{x}$ at time $t_{l}$, the reservation decision is made just before sending out the updated control packet, $C P_{x}$ ', i.e., at time $\left(t_{1}{ }^{\prime}-T_{0}\right)$, where $T_{0}$ is the transmission time of the control packet. The transmission time $T_{0}$ is hardware dependent. We can assume that it is a constant value at each node, and it is usually negligible compared to the duration of scheduling. The procedure of the proposed scheme is as follows: after receiving a control packet, the control unit at the node performs processing control packet. At time $t_{l}$, the scheduling decision is not really made, but virtually; i.e., the virtual scheduling decision does not reserve any network resources but marks the interval to indicate that the interval may be used for a data burst. Before the electronically buffered control packet is sent to the downstream node, the scheduling decision is really made at time $\left(t_{1}{ }^{\prime}-T_{0}\right)$. During the period from $t_{1}$ to $\left(t_{1}{ }^{\prime}-T_{0}\right)$, the channel assignment is based on the order of data burst arrival sequence. Since the control unit at node $i$ can gather more information from arriving control packets, a better scheduling decision may be made and the network resource can be used more efficiently. Since more information of the subsequent DBs is available to the node, better resource allocation can be achieved. Many sophisticated scheduling schemes of the DBs in FDLs are possible with the proposed scheme. In this paper, however, the DBs in the FDLs are scheduled only according to their actual arrival sequence to demonstrate the principle of the DRD scheme.

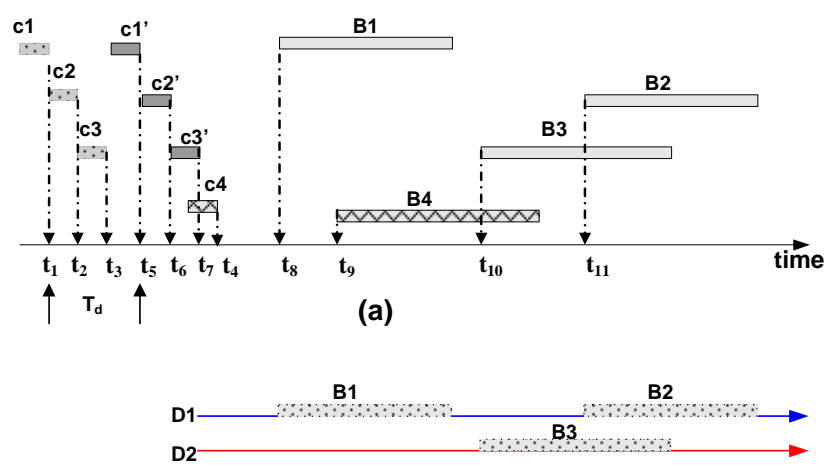

(b)

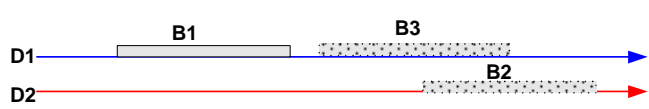

(c)

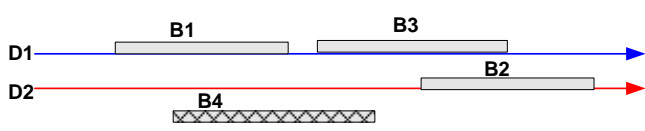

(d)

Figure 4. (a) The arrival and departure sequence of control packets and the corresponding data bursts, (b) at $t_{1}, B 1, B 2$ and $B 3$ are virtually scheduled at the output port, (c) at $\left(t_{1},-T_{0}\right), B 1, B 2$ and $B 3$ are re-scheduled according to the burst arrival sequence, and the ultimate scheduling decision of $B 1$ is made,

(d) the ultimate scheduling decisions of $B 1, B 2$ and $B 3$ when $C 4$ arrives.

Figure 4 shows that the proposed scheme can achieve better resource allocation than the general reservation scheme as illustrated in Figure 3. After processing $c l$ at $t_{l}, c l$ is electronically buffered until $t_{5}$ and its corresponding data burst $B 1$ is virtually scheduled on channel D1. c2 and c3 are 
processed in the same way. Thus, after $t_{3}, B 1, B 2$ and $B 3$ are virtually scheduled at the output port as shown in Figure 4(b). This virtual scheduling follows the arrival sequence of control packets and has a similar channel assignment as the general reservation scheme. At time $\left(t_{1},-T_{0}\right)$, ahead of sending the updated control packet $c l^{\prime}$ to the downstream node, the ultimate scheduling decision of $B 1$ is made. Based on the virtual scheduling, $B 1, B 2$ and $B 3$ are re-scheduled according to the burst arrival sequence. Therefore, $B 1$ is finally scheduled on channel $D 1, B 3$ is virtually scheduled on channel $D 1$ too and $B 2$ is virtually scheduled on channel $D 2$ as shown in Figure 4(c). The ultimate scheduling decision of $B 2$ and $B 3$ follows a similar process. Consequently, $B 1$ and $B 3$ are scheduled on $D 1$, and $B 2$ is scheduled on $D 2$. When $c 4$ arrives at the node, it can successfully schedule its data burst $B 4$ on channel $D 2$ without burst contention resolutions, as shown in Figure 4(d).

There are proposals for delaying the resource reservation to improve the system performance but the focus is on the output channel scheduling. Virtual Fixed Offset time (VFO) [11] is one such proposal. In VFO, each DB is assumed to be delayed by a fixed amount of time; therefore the node can schedule DBs according to their actual arrival times. However, to implement VFO, all input ports at each node must have an FDL module with a fixed delay. Since each DB needs to be buffered with the same delay, burst contention may not be effectively resolved. For the example shown in Figure 4, if the VFO reservation scheme is deployed, all four data burst are buffered with the same delay by the FDL at the input ports. Since $B 4$ is unnecessarily buffered and FDLs are not used to provide burst contention resolution, data burst $B 4$ is dropped. In the proposed delayed reservation scheme, FDLs are not only used to resolve burst contention but also to take advantage of the process of electronic buffering associated with the FDL delay to achieve more efficient resource allocation. In the following section, we compare the performance of the VFO approach and the delayed reservation decision scheme by simulations.

\section{PERFORMANCE EVALUATION}

We run simulations to investigate the network performance under the proposed DRD scheme in the NSFNET topology, as shown in Figure 5.

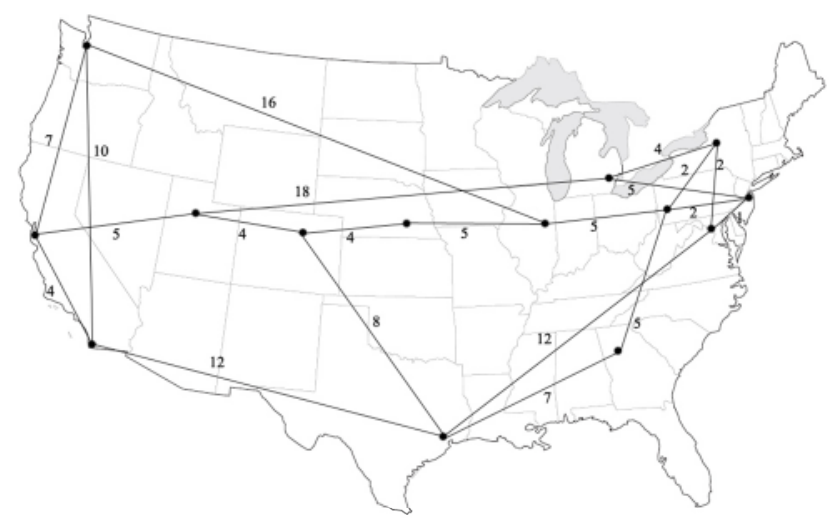

Figure 5. The NSFNET topology, 1991 (the original map is available at ftp.uu.net/inet/maps/nsfnet/)
Following the common simulation assumptions [11], the input network traffic is in the form of data bursts and follows the Poisson distribution. The data burst length is an exponential distribution. The average control packet processing time is assumed to be one tenth of the average data burst transmission time. We assume that each link has one dedicated wavelength as the control channel and ten wavelengths for carrying data bursts. Each node in the topology may be an edge node or a core node, and full wavelength conversion capability is available at each node. When a new data burst arrives at an edge node, it randomly chooses a destination from the rest of the nodes in the network and the shortest path routing algorithm is used. All nodes are assumed to receive the same offered load in the network.

Figure 6 compares the performance of the proposed DRD scheme with that of the existing OBS networks with optical buffers. The performance in loss probability of the general OBS with FDLs is plotted in dotted line; and that of DRD scheme is plotted in solid line. The lines with dots and crosses indicate that each node has an FDL with a fixed delay of 0.1 time units, those with circles and asterisks indicate that each node has an FDL with a fixed delay of 1 time unit, and those with squares and plus signs indicate that each node has an FDL module whose granularity is one and the maximum delay is 10 time units. We can observe that DRD has better performance. Although the DRD scheme requires the same hardware complexity, its delayed reservation decision can utilize resources more efficiently and therefore more DBs can be served.

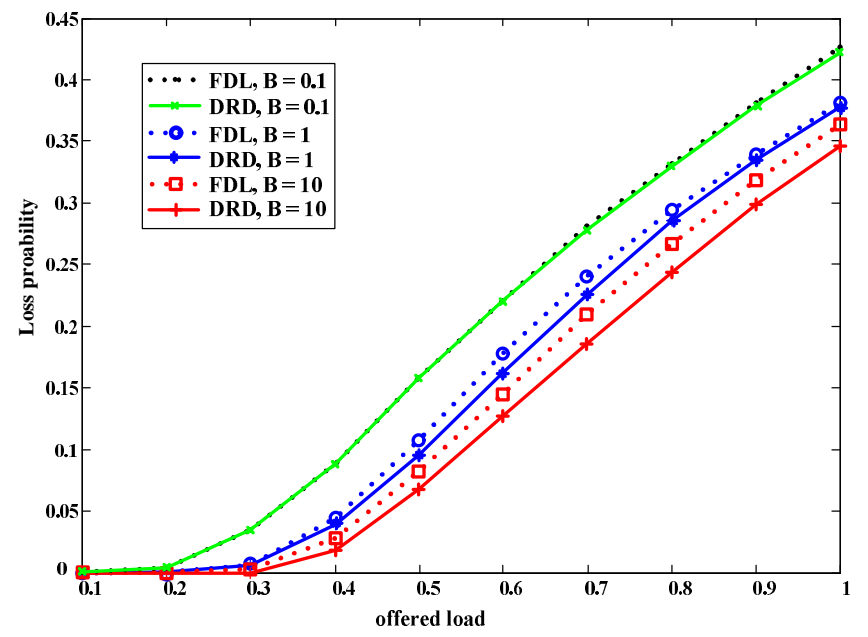

Figure 6. The loss probability of the general OBS with FDLs and the DRD scheme.

Figure 7 gives the performance comparison in loss probability of the delayed reservation decision scheme and the VFO scheme [11]. The VFO scheme, plotted in dotted lines, buffers each incoming data burst with a fixed delay, and thus the FDL share per link architecture must be deployed. The DRD scheme, deploying the FDL share per node architecture, is plotted in solid lines. The lines with squares and crosses indicate that each node has an FDL with a fixed delay of one time of the control packet processing time, i.e., 0.1 time units. Those with circles and asterisks indicate that each node has an FDL with a fixed delay of ten times of the control packet 
processing time. We observe that, only when the network load is high, will VFO scheme have lower burst loss probability. VFO uses FDL to delay data bursts, and the scheduler can resort data bursts according to their arrival times in order to utilize bandwidth more efficiently. When the network offered load is high, FDL buffers in both VFO and DRD are filled with data bursts. In VFO, the interval to optimize scheduling is fixed at $B$. In DRD, the interval is dependent on the delay required to resolve burst contention, which is less than $B$ in most cases. Therefore, VFO has lower burst loss probability when the network is heavily loaded. While the network is not highly loaded, for example when the load is less than 0.8 with buffer of one time unit delay, the DRD scheme has much lower loss probability than the VFO scheme. This is due to the fact that VFO buffers each incoming data burst and the burst contention may not be effectively resolved.

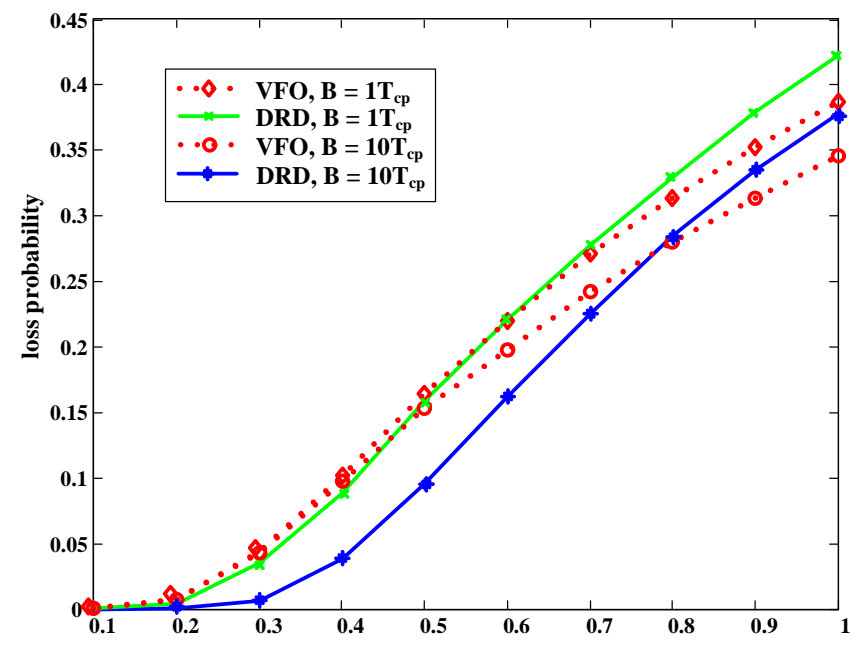

Figure 7. The loss probability of the general OBS with FDLs and the DRD scheme.

\section{CONCLUSIONS}

Based on existing OBS networks with optical buffers, we propose the delayed reservation decision scheme to enhance the network performance. The proposed scheme has the same hardware complexity at each node as the existing OBS networks with optical buffers, but allows extra electronic processing of control packets to utilize the network resources more efficiently. Compared to other delayed reservation schemes such as the virtual fixed offset time, the proposed DRD scheme decreases the hardware complexity, and improves the network performance in loss probability when the network is not highly loaded.

\section{ACKNOWLEDGMENT}

This research is supported in part by the Areas of Excellence Scheme established under the University Grants Committee of the Hong Kong Special Administrative Region, China (Project No. AoE/E-01/99). Additional support is provided by a grant from The Hong Kong Polytechnic University (Project Number A-PF98).

\section{REFERENCES}

[1] K. C. Lee and V. O. K. Li, "A wavelength-convertible optical network," Journal of Lightwave Technology, vol. 11, no. 5, pp. 962-970, 1993.

[2] X. C. Yuan, V. O. K. Li, C. Y. Li, and P. K. A. Wai, "A novel selfrouting address scheme for all-optical packet-switched networks with arbitrary topologies," Journal of Lightwave Technology, vol. 21, no. 2, pp. 329-339, 2003.

[3] M. Yoo and C. Qiao, "Just-Enough-Time (JET): A High Speed Protocol for Bursty Traffic in Optical Networks," in IEEE/LEOS Technologies for Globle Information Infrastructure 1997, pp. 26-27, 1997.

[4] C. Qiao and M. Yoo, "Optical burst switching (OBS) - a new paradigm for an optical Internet," Journal of High Speed Networks, vol. 8, no. 1, pp. 69-84, 1999.

[5] M. Yoo, M. Jeong, and C. Qiao, "A high-speed protocol for bursty traffic in optical networks," in SPIE Proceedings, All Optical Communication Systems: Architecture, Control and Network Issues, pp. 3230, 1997.

[6] C. Y. Li, G. M. Li, P. K. A. Wai, and V. O. K. Li, "Novel resource reservation schemes for optical burst switching," in Proceedings of IEEE ICC 2005, 3ed, pp. 1651-1655, 2005.

[7] Y. Xiong, M. Vandenhoute, and H. C. Cankaya, "Control architecture in optical burst-switched WDM networks," IEEE Journal on Selected Arears in Communications, vol. 18, no. 10, pp. 1838-1851, 2000.

[8] M. Yoo, C. Qiao, and S. Dixit, "The effect of limited fiber delay lines on QoS performance of optical burst switched WDM networks," in Proceedings of IEEE ICC 2002, 2ed, pp. 974-979, 2000.

[9] M. Yoo, C. Qiao, and S. Dixit, "QoS performance of optical burst switching in IP-over-WDM networks," IEEE Journal on Selected Arears in Communications, vol. 18, no. 10, pp. 2062-2071, Oct. 2000.

[10] X. Lu and B. L. Mark, "Performance modeling of optical-burst switching with fiber delay lines," IEEE Transactions on Communications, vol. 52, no. 12, pp. 2175-2183, 2004.

[11] J. Li, C. Qiao, J. Xu, and D. Xu, "Maximizing throughput for optical burst switching networks," in Proceedings of IEEE INFOCOM 2004, 3ed, pp. 1853-1863, 2004. 